\title{
ALGORITHMIC INVARIANTS FOR ALEXANDER MODULES
}

\author{
J. GAGO-VARGAS, M.I. HARTILLO-HERMOSO, AND J.M. UCHA-ENRÍQUEZ
}

\begin{abstract}
Let $G$ be a group given by generators and relations. It is possible to compute a presentation matrix of a module over a ring through Fox's differential calculus. We show how to use Gröbner bases as an algorithmic tool to compare the chains of elementary ideals defined by the matrix. We apply this technique to classical examples of groups and to compute the elementary ideals of Alexander matrix of knots up to 11 crossings with the same Alexander polynomial.
\end{abstract}

\section{INTRODUCTION}

Let $G=\langle\mathbf{x}: \mathbf{r}\rangle$ be a group given by generators and relations, where $\mathbf{x}=$ $\left(x_{1}, \ldots, x_{n}\right)$ is a base of the free group $F$ and $\mathbf{r}=\left(r_{1}, \ldots, r_{m}\right)$ are the relations. Through Fox's differential calculus [Crowell et al.(1977)] it is possible to compute the presentation matrix of the Alexander module of the group. We review briefly these concepts.

We build from $G$ the ring of the group $\mathbb{Z} G$. A derivation over the group ring is a map $D: \mathbb{Z} G \rightarrow \mathbb{Z} G$ such that

$$
\begin{gathered}
D\left(\nu_{1}+\nu_{2}\right)=D \nu_{1}+D \nu_{2}, \\
D\left(\nu_{1} \nu_{2}\right)=\left(D \nu_{1}\right) \mathfrak{t}\left(\nu_{2}\right)+\nu_{1} D \nu_{2},
\end{gathered}
$$

where $\mathfrak{t}$ is the trivializer and $\nu_{1}, \nu_{2} \in \mathbb{Z} G$. For elements in $G$, the second condition is

$$
D\left(g_{1} g_{2}\right)=D g_{1}+g_{1} D g_{2} .
$$

Then a derivation can be seen as the unique linear extension to $\mathbb{Z} G$ of a map $D: G \rightarrow \mathbb{Z} G$ that verifies the previous condition.

It is known that each generator $x_{j}$ in the group $G$ defines a unique derivation $D_{j}=\partial / \partial x_{j}$ in $\mathbb{Z} G$, such that

$$
\frac{\partial x_{i}}{\partial x_{j}}=\delta_{i j}
$$

Let $H$ be the abelianized group of $G$. Considering the group rings we have the composition of maps

$$
\mathbb{Z} F \stackrel{D_{j}}{\rightarrow} \mathbb{Z} F \stackrel{\gamma}{\rightarrow} \mathbb{Z} G \stackrel{\text { a }}{\rightarrow} \mathbb{Z} H,
$$

where $\gamma$ is the projection and $\mathbf{a}$ is the abelianizer. The Alexander matrix from $G$ is $A=\left(a_{i j}\right)$, where

$$
a_{i j}=\mathbf{a} \gamma\left(\frac{\partial r_{j}}{\partial x_{i}}\right)
$$

2000 Mathematics Subject Classification. Primary 13P10, 57M05; Secondary 57M27.

Key words and phrases. Gröbner bases, Elementary ideals, invariants of knots.

All authors partially supported by MTM2004-01165 and FQM-333. 
Note that this matrix is the transposed of the matrix defined by [Crowell et al.(1977)]. The Alexander matrix presents a module over the ring $\mathbb{Z} H$. If two groups are isomorphic then the modules are isomorphic.

A finite presentation for $M$ is an exact sequence

$$
R^{n} \stackrel{\alpha}{\rightarrow} R^{m} \stackrel{\Phi}{\rightarrow} M \rightarrow 0
$$

where $R^{n}$ and $R^{m}$ are free $R$-modules with respective bases $\mathbf{f}_{1}, \ldots, \mathbf{f}_{n}$ and $\mathbf{e}_{1}, \ldots \mathbf{e}_{m}$. If $\alpha$ is represented by the matrix $A$ with respect to these bases then the $m \times n$ matrix $A$ is a presentation matrix for $M$.

Theorem 1. [Lickorish(1998), Thm. 6.1] If $A_{1}$ and $A_{2}$ are presentation matrices of a module $M$ then they are related by a sequence of matrix transformations of the following form and their inverses:

(1) Permutation of rows and columns.

(2) Replacement of the matrix $A_{1}$ by $\left(\begin{array}{cc}A_{1} & 0 \\ 0 & 1\end{array}\right)$.

(3) Addition of an extra column of zeros to the matrix $A_{1}$.

(4) Addition of a scalar multiple of a row (column) to another row (column).

We say that $A_{1}$ and $A_{2}$ are Fitting equivalents.

Definition 1. Let $M$ be a $R$ module, with an $m \times n$ presentation matrix $A$. The $r$ th elementary ideal $F_{r}$ of $M$ is the ideal generated by all the $(m-r+1) \times(m-r+1)$ minors of $A$.

By convention, $F_{r}(M)=R$ when $r>m$ and $F_{r}(M)=0$ if $r \leq 0$. They form an ascending chain $F_{k}(M) \subset F_{k+1}(M)$. The elementary ideals are independent of the presentation matrix chosen to evaluate them.

\section{Algorithms in the Ring Group}

The ring $\mathbb{Z} H$ is commutative, because $H$ is an abelian group, and it has a special form.

Proposition 1. The ring $\mathbb{Z} H$ is isomorphic to $\mathbb{Z}\left[x_{1}^{ \pm}, \ldots, x_{n}^{ \pm}\right] / J$, where $J$ is the ideal generated by the relations $r_{1}, \ldots, r_{m}$ under commutativity.

Proof. Through the abelianizer, all the relations have the form $\prod x_{i}^{e_{i}}=1$, so $J$ is generated by the elements $\prod x_{i}^{e_{i}}-1$.

Corollary 1. There is an algorithm to compare ideals in $\mathbb{Z} H$.

Proof. Through the bijection between ideals in $\mathbb{Z}\left[x_{1}^{ \pm}, \ldots, x_{n}^{ \pm}\right] / J$ and ideals in $R=$ $\mathbb{Z}\left[x_{1}^{ \pm}, \ldots, x_{n}^{ \pm}\right]$that contains $J$, the problem is reduced to compare ideals in $R$. In this ring we can compute Gröbner bases [Sims(1994), Pauer et al.(1999)], or by the isomorphism $R \simeq \mathbb{Z}\left[x_{1}, \ldots, x_{n}, w\right] /\left\langle x_{1} \cdots x_{n} w-1\right\rangle$ [Adams et al.(1994)].

There is no known algorithm to decide whether two matrices present isomorphic modules. There are other invariants as the ideal row (column) class [Fox et al.(1964)] or the Nakanishi index [Kawauchi(1996)]. However we do not know algorithms to compute them and ad hoc arguments are needed to give their values for specific matrices [Fox et al.(1964), Kearton et al.(2003)]. 
Example 1. Let consider the groups given by the presentations

$D_{8}=\left\langle x, y \mid x^{4}=1, y^{2}=1, y x y^{-1}=x^{-1}\right\rangle, Q_{8}=\left\langle x, y \mid x^{4}=1, x^{2}=y^{2}, y^{-1} x y=x^{-1}\right\rangle$.

$D_{8}$ is the dihedral group of order 8 (symmetry group of the square) and $Q_{8}$ is the quaternion group. A classical exercise in group theory is to show that these two groups are not isomorphic. Let see how can this be accomplished with elementary ideals. Let $r_{i}$ be the the relations in $D_{8}$ :

$$
r_{1}: x^{4}=1, r_{2}: y^{2}=1, r_{3}: y x y^{-1} x=1 \text {. }
$$

Then

$$
\begin{array}{lll}
\frac{\partial r_{1}}{\partial x}=1+x+x^{2}+x^{3}, & \frac{\partial r_{2}}{\partial x}=0, & \frac{\partial r_{3}}{\partial x}=y+x^{-1} \\
\frac{\partial r_{1}}{\partial y}=0, & \frac{\partial r_{2}}{\partial y}=1+y, & \frac{\partial r_{3}}{\partial y}=1-x^{-1}
\end{array}
$$

In the abelianized group we add the relation $x y=y x$, so $x^{2}=1, y^{2}=1$. The Alexander module of the group has a presentation matrix

$$
M\left(D_{8}\right)=\left(\begin{array}{ccc}
2+2 x & 0 & x+y \\
0 & y+1 & 1-x
\end{array}\right),
$$

over the $\operatorname{ring} \mathbb{Z}\left[x^{ \pm}, y^{ \pm}\right] /\left\langle x^{2}-1, y^{2}-1\right\rangle$.

We proceed in an analogous way with $Q_{8}$. We write the relations

$$
s_{1}: x^{4}=1, s_{2}: x^{2} y^{-2}=1, s_{3}: x y^{-1} x y=1,
$$

and

$$
\begin{array}{lll}
\frac{\partial s_{1}}{\partial x}=1+x+x^{2}+x^{3}, & \frac{\partial s_{2}}{\partial x}=1+x, & \frac{\partial s_{3}}{\partial x}=1+x y^{-1} \\
\frac{\partial s_{1}}{\partial y}=0, & \frac{\partial s_{2}}{\partial y}=-x^{2}(1+y), & \frac{\partial s_{3}}{\partial y}=-x y^{-1}+y^{-1}
\end{array}
$$

As before, in the abelianized group the relations are reduced to $x^{2}=1, y^{2}=1$ and a presentation matrix of the Alexander module is

$$
M\left(Q_{8}\right)=\left(\begin{array}{ccc}
2+2 x & 1+x & 1+x y \\
0 & -1-y & -x y+y
\end{array}\right)
$$

over the $\operatorname{ring} \mathbb{Z}\left[x^{ \pm}, y^{ \pm}\right] /\left\langle x^{2}-1, y^{2}-1\right\rangle$.

We compute a Gröbner basis in $\mathbb{Z}\left[x^{ \pm}, y^{ \pm}\right]$of the second elementary ideal. Adding the polynomials $x^{2}-1, y^{2}-1$, we get

$$
F_{2}\left(M\left(D_{8}\right)\right)=\langle 4,1+y, 1-x\rangle, F_{2}\left(M\left(Q_{8}\right)\right)=\langle 2,1+x, 1+y\rangle .
$$

They are different so the groups are not isomorphic.

Example 2. In [Kanenobu(1986)] it is defined a class of knots $K_{p, q}$, with $p, q \in \mathbb{N}$, that has the Alexander matrix

$$
A_{p, q}=\left(\begin{array}{cc}
t^{2}-3 t+1 & (p-q) t \\
0 & t^{2}-3 t+1
\end{array}\right) .
$$

Lemma 2 of $[\operatorname{Kanenobu}(1986)]$ asserts that $K_{p, q}$ and $K_{p^{\prime}, q^{\prime}}$ have isomorphic Alexander modules if and only if $|p-q|=\left|p^{\prime}-q^{\prime}\right|$. Let us show how to apply our approach to give a new proof of this lemma. If the modules are isomorphic then the second elementary ideals $F_{2}$ must coincide. A Gröbner basis of the ideal is equal to $\left\{t^{2}-3 t+1, p-q\right\}$, so $F_{2}\left(A_{p, q}\right)=F_{2}\left(A_{p^{\prime}, q^{\prime}}\right)$ if and only if $|p-q|=\left|p^{\prime}-q^{\prime}\right|$. 


\section{An APPlication to KNOT THEORY}

One of the main invariants in knot theory is the fundamental group of the knot complement. The Alexander matrix can be computed from the Seifert matrix, and with the tables listed in [Burde et al.(1985)] and [Livingston(2004)] we can get the Alexander matrix of knots up to 11 crossings. As an application of the algorithm described before we give a list of knots with the same Alexander polynomial (grouped by boxes) and where the elementary ideals give more information to distinguish knots (see Table 1). For example, from Table 1 we deduce that $11 a_{102}$ and $11 a_{181}$ are different, but we cannot say anything about $11 a_{102}$ and $11 a_{199}$.

The first step was to compute the Alexander matrix of the knot and reduce it through the transformations given by Theorem 1 . In all cases we have got at most a $2 \times 2$ presentation matrix, so $F_{3}$ is always equal to $R$. The Gröbner bases were computed over the ring $\mathbb{Z}[t, w]$, adjoining to the ideals the polynomial $t w-1$.

\section{REFERENCES}

Adams et al.(1994). W.W. Adams, P. Loustaunau, An introduction to Gröbner bases, volume 3 of Graduate Studies in Mathematics, American Mathematical Society, Providence, RI, 1994.

Burde et al.(1985). G. Burde, H. Zieschang, Knots, volume 5 of de Gruyter Studies in Mathematics, Walter de Gruyter, Berlin and New York, 1985.

Crowell et al.(1977). R.H. Crowell, R.H. Fox, Introduction to Knot Theory, volume 57 of Graduate Texts in Mathematics, Springer-Verlag, New York, 1977.

Fox et al.(1964). R.H. Fox, N. Smythe, An ideal class invariant of knots, Proc. Amer. Math. Soc., 15:707-709, 1964.

Kanenobu(1986). T. Kanenobu, Infinitely many knots with the same polynomial invariant, Trans. Amer. Math. Soc., 97:158-162, 1986.

Kawauchi(1996). A. Kawauchi, A survey of knot theory, Birkhäuser Verlag, Basel, 1996.

Kearton et al.(2003). C. Kearton, S.M.J. Wilson, Knot modules and the Nakanishi index, Proc. Amer. Math. Soc., 131:655-663, 2003.

Lickorish(1998). W.B.R. Lickorish, An introduction to knot theory, volume 175 of Graduate Texts in Mathematics, Springer-Verlag, New York, 1998.

Livingston(2004). C. Livingston, Table of knot invariants at http://www. indiana.edu/ ${ }^{k n o t i n f o / . ~}$

Pauer et al.(1999). F. Pauer, A. Unterkircher, Gröbner Bases for Ideals in Laurent Polynomials Rings and their Application to Systems of Difference Equations, Appl. Algebra Engrg. Comm. Comput., 9:271-291, 1999.

Sims(1994). C.C. Sims, Computation with finitely presented groups, volume 48 of Encyclopedia of Mathematics and its Applications, Cambridge University Press, Cambridge, 1994.

Depto. de Álgebra, Universidad de Sevilla. Apdo. 1160, E-41080 Sevilla (Spain)

E-mail address: gago@us.es

Depto. de Matemáticas, Universidad de Cádiz. Apdo. 40, E-11510 Puerto Real (Spain)

E-mail address: isabel.hartillo@uca.es

Depto. de Álgebra, Universidad de Sevilla. Apdo. 1160, E-41080 Sevilla (Spain)

E-mail address: ucha@us.es 
TABLE 1. Elementary ideals

\begin{tabular}{|l|l|}
\hline Name & Elem. ideal $F_{2}$ \\
\hline $11 n_{100}$ & $R$ \\
$9_{37}$ & $\langle 3, t-2\rangle$ \\
& \\
\hline $11 n_{97}$ & $R$ \\
$6_{1}$ & $R$ \\
$9_{46}$ & $\langle 3, t+1\rangle$ \\
& \\
\hline $11 a_{102}$ & $R$ \\
$11 a_{181}$ & $\langle 3, t-2\rangle$ \\
$11 a_{199}$ & $R$ \\
& \\
\hline $10_{113}$ & $R$ \\
$11 a_{107}$ & $\left\langle 2, t^{2}+t+1\right\rangle$ \\
$11 a_{347}$ & $\left\langle 2, t^{2}+t+1\right\rangle$ \\
& \\
\hline $11 a_{187}$ & $R$ \\
$11 a_{249}$ & $\langle 3, t-2\rangle$ \\
$11 a_{38}$ & $R$ \\
$11 a_{8}$ & $R$ \\
& \\
\hline $11 a_{132}$ & $\left\langle 2, t^{2}+t+1\right\rangle$ \\
$11 a_{352}$ & $\left\langle 3, t^{2}-t+1\right\rangle$ \\
$11 a_{6}$ & $R$ \\
& \\
\hline $10_{65}$ & $\left\langle 2, t^{2}+t+1\right\rangle$ \\
$10_{77}$ & $R$ \\
$11 n_{71}$ & $\left\langle-t^{2}+t-1\right\rangle$ \\
$11 n_{75}$ & $\left\langle-t^{2}+t-1\right\rangle$ \\
& \\
\hline $10_{103}$ & $\langle 5, t+1\rangle$ \\
$10_{40}$ & $R$ \\
\hline & \\
& \\
\hline
\end{tabular}

\begin{tabular}{|l|l|}
\hline Name & Elem. ideal $F_{2}$ \\
\hline $10_{140}$ & $\left\langle 2, t^{2}-t+1\right\rangle$ \\
$11 n_{73}$ & $\left\langle t^{2}-t+1\right\rangle$ \\
$11 n_{74}$ & $\left\langle t^{2}-t+1\right\rangle$ \\
& \\
\hline $11 n_{116}$ & $R$ \\
$11 n_{49}$ & $\left\langle 2, t^{2}+t+1\right\rangle$ \\
& \\
\hline $11 n_{1}$ & $R$ \\
$9_{48}$ & $\langle 3, t+1\rangle$ \\
\hline $10_{155}$ & $\langle t+1,5\rangle$ \\
$11 n_{37}$ & $R$ \\
$8_{9}$ & $R$ \\
& \\
\hline $1 n_{164}$ & $\left\langle t^{2}-t+1\right\rangle$ \\
$11 n_{85}$ & $R$ \\
$8_{18}$ & $\left\langle t^{2}-t+1\right\rangle$ \\
$9_{24}$ & $R$ \\
& \\
\hline $10_{163}$ & $\left\langle 2, t^{2}+t+1\right\rangle$ \\
$11 n_{87}$ & $R$ \\
$9_{28}$ & $R$ \\
$9_{29}$ & $R$ \\
\hline $10_{59}$ & $R$ \\
$11 n_{66}$ & $R$ \\
$9_{40}$ & $\left\langle t^{2}-3 t+1\right\rangle$ \\
\hline $10_{42}$ & $R$ \\
$10_{75}$ & $\langle 3, t+1\rangle$ \\
& \\
\hline
\end{tabular}

\begin{tabular}{|l|l|}
\hline Name & Elem. ideal $F_{2}$ \\
\hline $10_{60}$ & $R$ \\
$11 n_{165}$ & $\left\langle 2, t^{2}+t+1\right\rangle$ \\
\hline $11 a_{223}$ & $R$ \\
$11 n_{148}$ & $\left\langle 5, t^{2}+2 t+1\right\rangle$ \\
& \\
\hline $11 a_{108}$ & $R$ \\
$11 a_{139}$ & $R$ \\
$11 a_{231}$ & $\left\langle t^{2}-t+1\right\rangle$ \\
$11 a_{57}$ & $\left\langle t^{2}-t+1\right\rangle$ \\
$11 a_{88}$ & $R$ \\
& \\
\hline $11 a_{109}$ & $R$ \\
$11 a_{44}$ & $\left\langle t^{2}-t+1\right\rangle$ \\
$11 a_{47}$ & $\left\langle t^{2}-t+1\right\rangle$ \\
& \\
\hline $10_{123}$ & $\left\langle t^{4}-3 t^{3}+3 t^{2}-3 t+1\right\rangle$ \\
$11 a_{28}$ & $R$ \\
\hline $10_{87}$ & $R$ \\
$10_{98}$ & $\left\langle 1-t+t^{2}\right\rangle$ \\
$11 a_{165}$ & $\left\langle 2,1-t+t^{2}\right\rangle$ \\
$11 a_{58}$ & $R$ \\
$11 n_{72}$ & $\left\langle 1-t+t^{2}\right\rangle$ \\
\hline $10_{144}$ & $\left\langle 2,1-t+t^{2}\right\rangle$ \\
$11 n_{99}$ & $R$ \\
\hline $11 n_{83}$ & $\left\langle 2, t^{2}+t+1\right\rangle$ \\
941 & $\langle 7,1+t\rangle$ \\
\hline
\end{tabular}




\begin{tabular}{|l|l|}
\hline Name & Elem. ideal $F_{2}$ \\
\hline $11 n_{162}$ & $\left\langle 2, t^{2}+t+1\right\rangle$ \\
$9_{39}$ & $R$ \\
& \\
\hline $11 a_{31}$ & $R$ \\
$11 a_{317}$ & $\langle t+1,5\rangle$ \\
& \\
\hline $10_{67}$ & $R$ \\
$10_{74}$ & $\langle 3, t+1\rangle$ \\
$11 n_{68}$ & $R$ \\
& \\
\hline $11 a_{157}$ & $\left\langle 2, t^{4}+t^{2}+1\right\rangle$ \\
$11 a_{264}$ & $R$ \\
$11 a_{305}$ & $R$ \\
$11 a_{80}$ & $R$ \\
& \\
\hline $10_{63}$ & $\left\langle 2, t^{2}+t+1\right\rangle$ \\
$9_{38}$ & $R$ \\
& \\
\hline $11 a_{277}$ & $\langle t+1,3\rangle$ \\
$11 a_{99}$ & $R$ \\
\hline $11 a_{196}$ & $\langle 7, t+1\rangle$ \\
$11 a_{216}$ & $R$ \\
$11 a_{286}$ & $R$ \\
& \\
\hline & \\
\hline
\end{tabular}

